\title{
Isolation rates and antimicrobial sensitivity patterns of bovine mastitis pathogens in peri-urban area of Nairobi, Kabete, Kenya
}

\author{
G. K. Gitau ${ }^{1 *}$, J. K. Wabacha ${ }^{1}$, C. M. Mulei ${ }^{1}$, S. Ndurumo ${ }^{1}$ and J. M. Nduhiu ${ }^{1}$ \\ ${ }^{1}$ Department of Clinical Studies, Faculty of Veterinary Medicine, University of Nairobi, P.O. Box \\ 29053-00625, Nairobi, Kenya \\ *Corresponding author (G. Gitau): gkgitau@uonbi.ac.ke
}

\begin{abstract}
A retrospective study was carried out to determine the prevalence and antimicrobial sensitivity to commonly occurring bovine mastitis pathogens in the peri-urban area of Nairobi between 1994 and 2004. Samples were mainly drawn from the area covered by the ambulatory services of the Faculty of Veterinary Medicine, University of Nairobi, Veterinary Clinic in Kabete, Nairobi.A total of 888 isolates were studied from bacterial cultures obtained from the bovine samples collected at the clinic. The most common microbial isolates were Streptococcus species (20.6\%), Staphylococcus species (20.1\%), Escherichia coli (16.8\%), Klebsiella species (15.4\%), Actinomyces (13.9\%) and Pseudomonas species (8.3\%). The study showed that sensitivity to antimicrobial agents was highest for gentamycin and kanamycin while it was moderate to low for penicillin, ampicillin, tetracycline, streptomycin, amoxicillin and penicillin-dihydrostreptomycin. The results of the study however did not show any clear trend in sensitivity to antimicrobial agents over the 11-years period.
\end{abstract}

Keywords: bovine, mastitis, pathogens, antibacterial sensitivity, Kenya

\section{Introduction}

Bovine mastitis is the most common disease in dairy herds and is responsible for enormous economic losses to milk producers and milk processors due to reduced milk production, discarded milk and cost of replacement and treatment (Radostitis, 2001). Mastitis, which is the inflammation of the mammary gland, primarily results from invasion and colonization of the secretor tissue by several microbial organisms (Radostitis, 2001). The leading causes of mastitis are Staphylococcus aureus, Streptococcus agalactiae, Streptococcus uberis, Staphylococcus dysagalactiae, Escherichia coli and Corynebacerium bovis (Radostitis, 2001; Miltenburg et al., 1996; Barkema et al., 1999; Erskine et al., 2002 and Gitau et al., 2003). 
The success of bovine mastitis therapy depends on the aetiology, clinical presentation, and antimicrobial susceptibility of the aetiological agent among other factors (Miltenburg et al., 1996). Therapy failure in the management of mastitis could result from pathological changes that occur in the udder, aetiology related factors, pharmacokinetic properties of the antimicrobial drugs, poor animal husbandry and inadequate veterinary services. However, the control of mastitis has been successfully achieved through the establishment of effective herd health control programs (Erskine et al., 2002). Antimicrobial agents are the main therapeutic tools for the treatment and control of mastitis. Among the main reasons of low efficacy of antibiotic treatment of mastitis cases is the resistance of the bacteria to antimicrobials. Recently, several studies have been conducted to determine the antibacterial susceptibility patterns of mastitis pathogens isolated from clinical studies or submitted to diagnostic laboratories (Brown and Scasserra, 1990; Fang et al., 1996; De Olivera et al., 2000; Gitau et al., 2003; Haile, 2004). However, in Kenya little information exists as regards the antimicrobial sensitivity patterns of bacterial pathogens causing mastitis (Agumbah et al., 1983).

The purpose of the study was to determine the occurrence rate of bacterial pathogens causing mastitis and evaluate the antimicrobial resistance of mastitis causing pathogens in the peri-urban area of Nairobi.

\section{Materials and methods}

\section{Study Area}

The study was carried out in the peri-urban area of Nairobi, Kenya, which is served by the Ambulatory Clinic of the Faculty of Veterinary Medicine, University of Nairobi. The majority of the farmers in the area are smallholder and animals are confined in stalls all year round with feed being delivered to them though a few large scale dairy farmers are also present. The area has two rainy seasons and has an altitude of between 1500-2000 masl. The long rains occur between March and May while short rains occur between October and December.

\section{Sample Collection in the Field}

Quarter milk samples were collected by clinicians from clinical cases and subclinical cases that were positive for CMT. For clinical cases that had obvious clots in milk, a CMT was not performed. Samples collected by the Ambulatory Clinic during the period 1994 to 2004 were analysed. The teat ends were 
cleaned with alcohol swabs and allowed to dry. The first few streams were discarded and then about $5 \mathrm{ml}$ of secretion was collected in sterile universal bottles. The samples were submitted to the Microbiology Diagnostic Laboratory of the Department of Clinical Studies, University of Nairobi for bacteriologic culture.

\section{Bacterial Culture and Identification}

The samples were inoculated by streaking onto the surface of $5 \%$ sheep blood agar and McConkey agar, and incubated at $37^{\circ} \mathrm{c}$ for $18-24 \mathrm{hrs}$. The plates were then examined for growth of micro-organisms with those having none re-incubated further for up to 4 days after which the conclusion of no growth of microorganisms was arrived at. Those having growth were studied macroscopically for both abundance and colonial morphology. The cultures were then examined microscopically for gram reaction and morphology. The targeted organisms during culture were mainly: Staphylococci spp., Streptococci spp., Escherichia coli, Klebsiella spp., Pseudomonas spp, Corynebacterium pyogenes and Proteus spp. The details on the procedure are as described by Holt et al., (1993).

All gram negative rods were divided into lactose and non-lactose fermenters. The lactose fermenters were subjected to a panel of 4 biochemical tests namely: Methyl-Red, Voges-Proskauer, Indole and Citrate fermentation, to determine whether they were E. coli or Klebsiella. The non-lactose fermenting Gram negative Rods were subjected to the following tests that is Oxidase Urease, Sacrose fermentation, and motility (Holt et al., 1993).

Those motile and oxidase positive were identified as Pseudomonas while the motile, oxidase negative and urease positive were identified as Proteus spp. The short uniformly staining rods that tested positive for catalase were identified as Corynebacteria while the slender, curved, barred or beaded rods that tested negative for catalase were identified as Corynebacterium pyogenes (Holt et al., 1993).

The small-medium sized colonies that were hemolytic or non-hemolytic on 5\% sheep blood agar and yielding gram positive cocci were subjected to catalase and coagalase tests. All the colonies that tested negative for catalase were identified as Streptococci. Those that tested positive for catalase were further tested with rabbit plasma for coagulase activity and those found to be positive were confirmed to be Staphylococus aureus (Holt et al., 1993). 


\section{Antimicrobial Sensitivity Tests}

The bacterial isolates were tested for sensitivity through a panel of 8 antimicrobial drugs that were commonly used by the Ambulatory clinic using Agar Disk Diffusion Technique (Mast Laboratories, UK). Commercially available drug impregnated paper disks either singly or combined as in Multodisk ${ }^{\circledR}$ (Oxoid), Mastring-S® (Mast Laboratories, UK) and Octodics ${ }^{\circledR}$ (HI Media) were applied onto the surface of sheep blood agar inoculated uniformly with the pathogen under test, then incubated overnight at $37^{\circ} \mathrm{C}$ (Holt et al., 1993). The effectiveness of a drug was determined by measuring the diameter of the zone of inhibition around the disc, the larger the diameter the more effective the drug. A scale of 0-3 was used to score the relative efficacy of various drugs against a given pathogen as presented below (Holt et al., 1993).

A zone diameter of $0-8 \mathrm{~mm}$ scored 0 or ' $\mathrm{R}$ ' for resistant

A zone diameter of 9-15 scored + or slightly sensitive

A zone diameter of $16-22 \mathrm{~mm}$ scored ++ or sensitive

A zone diameter of 23 and above scored +++ or very sensitive

\section{Data Management and Analysis}

All the isolates were first summarized according to the frequency of isolation and the proportion of the isolate from the overall total. Thereafter, data were summarized according to annual frequencies and proportions over the 11 year period for each isolate. Due to variations in the drug sensitivity disks available during the study period, some drug sensitivities were not carried out during certain years and these entries were noted as not tested (nt).

The isolates were further categorized and analysed as the proportions of isolates sensitive to each of the 8 antimicrobial drugs for tested for each year and then the overall total for the 11-year period. For the purpose of making statistical sense, the proportions (\%) that were included for comparisons were for those with 9 or more isolates per year (to avoid having too few cases reclassified further) while the trends were compared for those whose observations were made for more than 6 years. An attempt was also made to determine the analysis of trend using the same criterion above. 


\section{Results}

A total of 888 isolates were isolated, identified, subjected to antimicrobial sensitivity testing using the disk diffusion method. The most common microbial isolates were Streptococcus species (20.6\%), Staphylococcus species (20.1\%), Escherichia coli (16.8\%), Klebsiella species (15.4\%), Actinomyces (13.9\%) and Pseudomonas Species (8.3\%) (Table1).

The annual proportions of $E$. coli isolates that were sensitive to Gentamycin and Kanamycin were the highest (over 90\% and 70\% respectively) (Table 2). The sensitivity trend did not show any decline or increase during the 11 year period. The annual proportions of $E$. coli isolates that were susceptible to Ampicillin, Teracycline and Streptomycin were generally average and showed a general decline over the years though this was not obviously consistent.

The annual proportions of Streptococci isolates were moderately sensitive to all the antimicrobials except Ampillicn where the sensitivity was over $60 \%$ except one year (Table 3). The other antimicrobials, that is Penicillin, Gentamycin, Teracycline, Streptomycin, Amoxycillin and Kanamycin showed major variations in sensitivity to the isolates and this ranged from high (over $80 \%$ ) to lower than below $20 \%$. The above variations did not reflect a specific trend pattern of either decline or rise during the 11 year period.

The annual proportions of Saphylococcus aureus isolates showed major inconsistencies in variations to the various antimicrobials but were moderately sensitive (over 60\%) to gentamycin, kanamycin, ampicillin and penicillindihydrostreptomycin but they were less sensitive to penicillin, tetracycline and streptomycin with a sensitivity generally below $60 \%$ (Table 4 ). There was no clear trend in rise or decline in the sensitivity pattern over the 11-year period.

The annual proportions of Klebsiella spp. isolates showed very high sensitivity to gentamycin and kanamycin (over $80-100 \%$ ) but moderate to low sensitivities to the other antimicrobials such as ampicillin, penicillin-dihydrostreptomycin, tetracycline and streptomycin (Table 5). There was no clear trend in rise or decline in the sensitivity pattern over the 11-year period.

The annual proportions of Actinomyces spp. isolates showed a general moderate to high sensitivity to the various antimicrobials with high sensitivities observed for penicillin, ampicillin, gentamycin and penicillin-dihydrostreptomycin and moderate sensitivities for kanamycin, tetracycline and streptomycin (Table 6). 
There was no clear trend in rise or decline in the sensitivity pattern over the 11-year period.

Table 1: Bacterial isolates and their frequency of occurrence from Kabete area of Kenya in the period $1994-2004$

\begin{tabular}{lcc}
\hline Organisms & Number of isolates & Rate of isolation \\
\hline Escherichia coli & 149 & 16.8 \\
Streptococcus species & 183 & 20.6 \\
Staphylococcus aureus & 179 & 20.2 \\
Klebsiella species & 137 & 15.4 \\
Pseudomonas & 74 & 8.3 \\
Actinomyces $^{\text {Others }}$ & 123 & 13.9 \\
Total $^{\mathrm{a}}$ & 43 & 4.8 \\
\hline
\end{tabular}

aOthers include Norcardia, Proteus, Pasteurella, Yeast and Salmonella spp

\section{Discussion}

The results of the study showed that the important bacterial organisms isolated in the peri-urban area of Nairobi Kenya were Streptococcus species, Staphylococcus aureus and Escherichia coli as has been reported in other studies (Barkema et al., 1999; Radostitis, 2001; Erskine et al., 2002; Gitau et al., 2003; Haile, 2004). The other moderately prevalent isolates were Klebsiella spp., Actinomyces and Pseudomonas spp. A study carried out in the same area about 25 years ago showed that $E$. coli was the most prevalent $(78 \%)$ of the coliform mastitis although it contributed to about $29 \%$ of the total isolates (Agumbah et al., 1983For other non-severe infections, the farmers may either not notice the problem (in sub-clinical cases) or may try to manage through off-the-counter purchase of intramammary formulations. Another more recent study carried out in Zanzibar showed that Staphylococcus and Streptococcus spp were the most prevalent in the smallholder dairy farms (Gitau et al., 2003). The latter study was based on milk samples collected from both sub-clinical (CMT positive) and clinical cases.

Although the antimicrobial sensitivity pattern from this study tended to show some similarity from other studies carried out elsewhere, comparison with other studies must be made cautiously because of the differences in sensitivities of assays, selection of cows for milk samples, bacteriological culture and identification and regional differences in pathogen populations as noted earlier (Myllys et al., 1998, Erskine et al., 2001). The results in this 


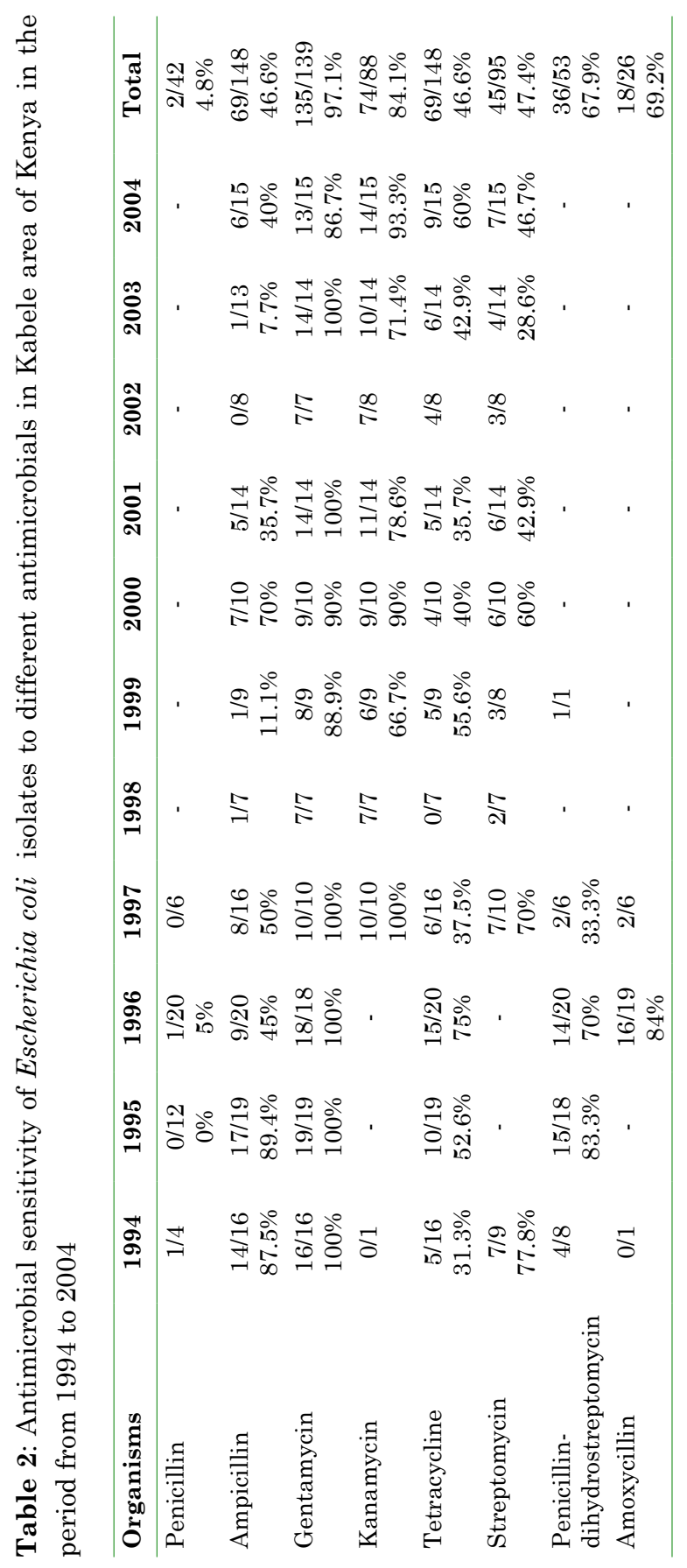

Ethiop. Vet. J., 2011, 15 (1), 1-13 


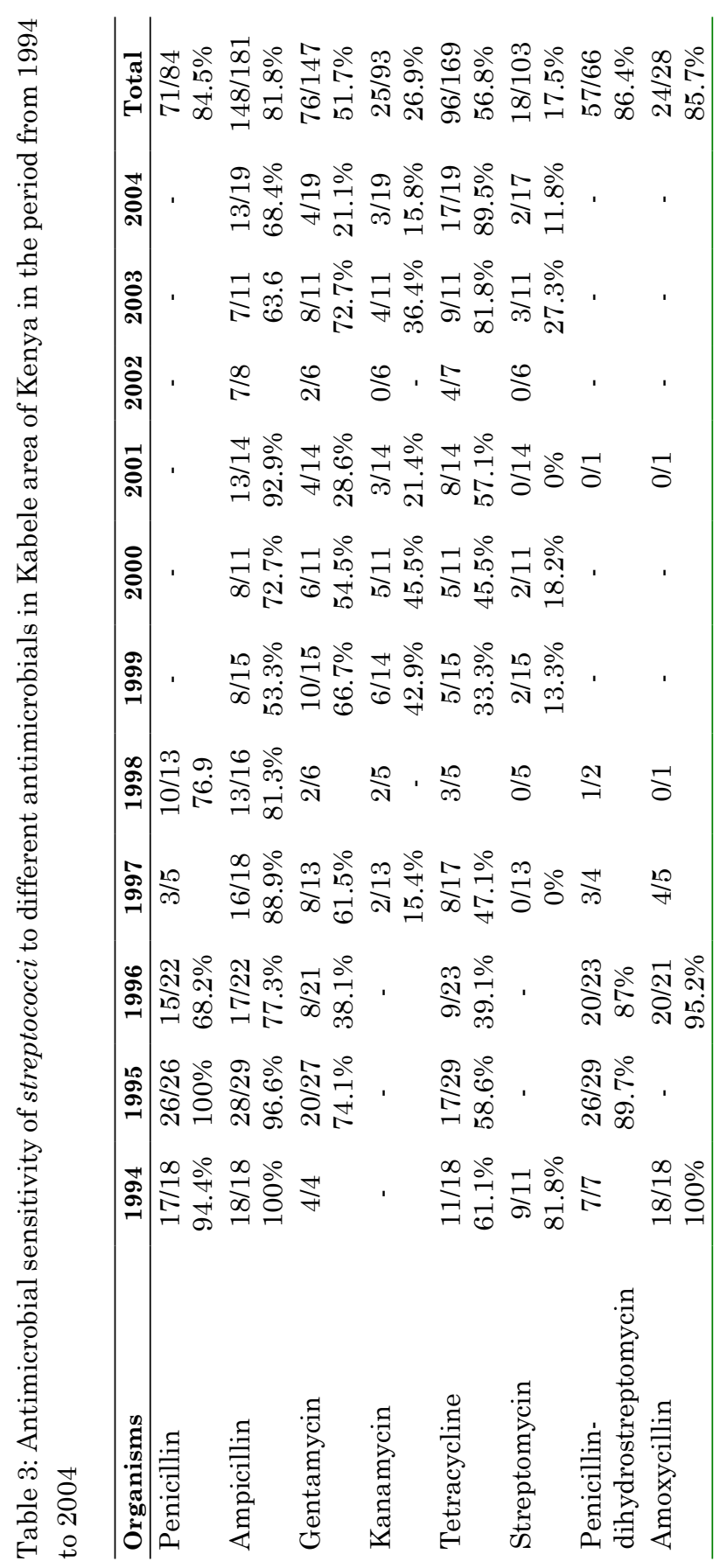

Ethiop. Vet. J., 2011, 15 (1), 1-13 


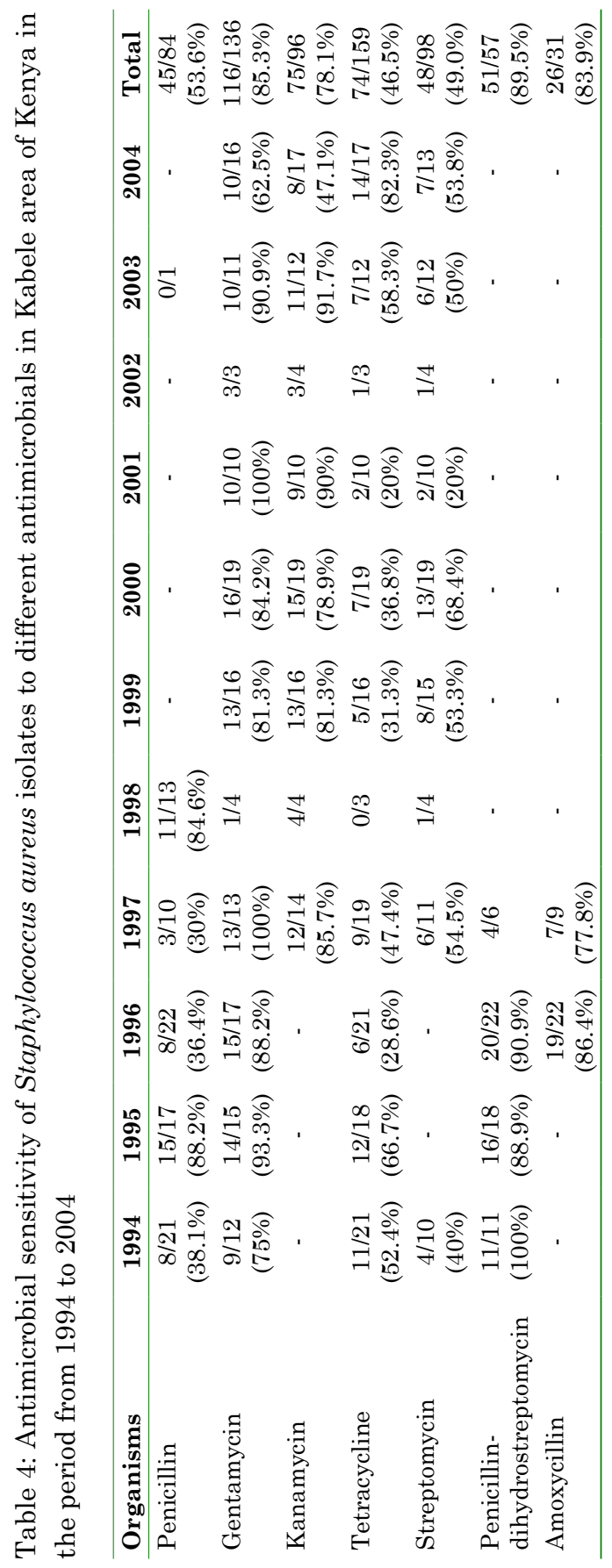

Ethiop. Vet. J., 2011, 15 (1), 1-13 


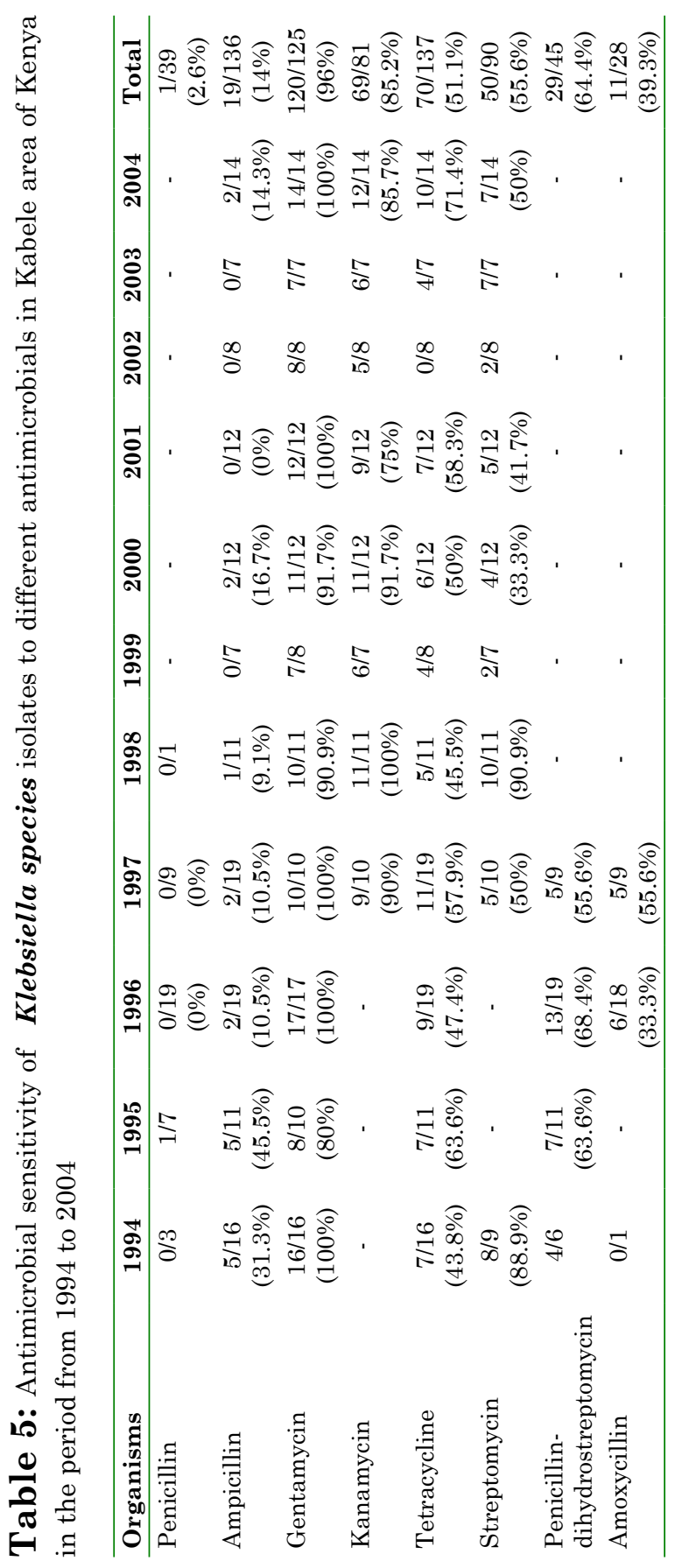




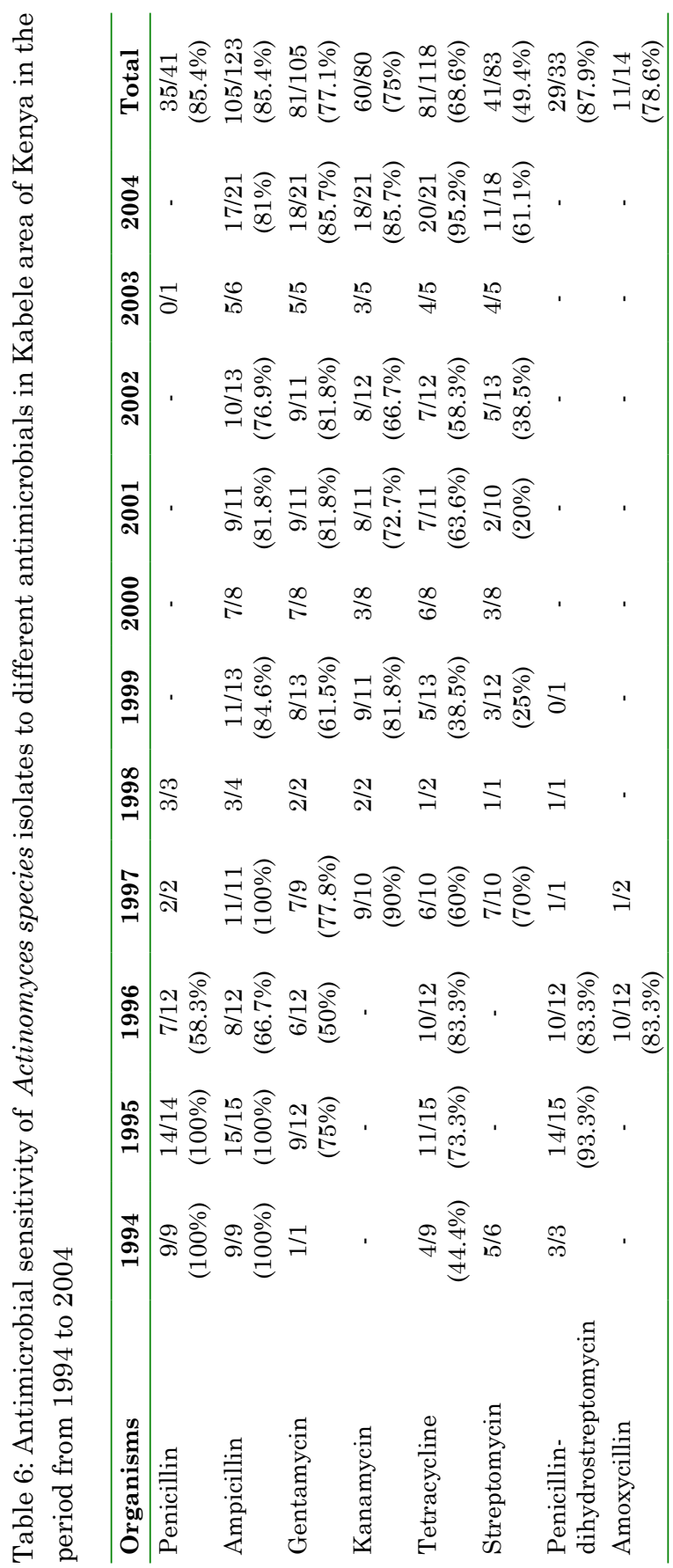

Ethiop. Vet. J., 2011, 15 (1), 1-13 
study showed that the proportion of sensitive pathogens was generally higher for gentamycin and kanamycin and moderate to low for the other antibacterial drugs. This can be explained by the fact that there has been extensive use and misuse, and availability of penicillin and their derivatives, and tetracyclines for many years in the area while gentamycin and kanamycin were introduced recently. The low sensitivity to the latter two may not therefore have developed extensively. Some studies have shown different results, for example, a low sensitivity to drugs routinely used for S. aureus mastitis therapy in 11 different countries was reported earlier (De Olivera et al., 2000). In another study, Streptococcal strains were reported to show low sensitivity to oxytetracyclines and streptomycin compared to penicillin (Brown and Scasserra, 1990). A 7-year Finnish study showed a non-decreasing sensitivity to various antimicrobiols to the various mastitis pathogens apart from penicillin on S. uberis (Erskine et al., 2001).

The study showed that Streptococcus species, Staphylococcus aureus and Escherichia coli still remain the most important causes of bovine mastitis in the peri-urban area of Nairobi, Kenya. The study further concludes that there is clear evidence of antimicrobial resistance to the most commonly used drugs by some of the mastitis pathogens in the study area. The increasing incidence of the use of non-efficacious and sub-therapeutic regimes of antibacterials especially in developing countries by all cadres of para-veterinarians and quacks remains to be an important concern. We recommend that a welldesigned epidemiologic study be carried out to establish a better picture of antibacterial resistance to various mastitis pathogens in the peri-urban area of Nairobi and other intensive dairy farming areas of Kenya in order to advice on the best therapeutic regime. In the meantime, veterinarians should avoid the use of the antimicrobials showing resistance to pathogens in the study area.

\section{Acknowledgements}

The authors would like to acknowledge all the Clinicians from the Department of Clinical Studies, University of Nairobi who participated in the collection of milk samples during the 10 year period. We would also like to thank the technical staff of the bacteriology laboratory of the Department of Clinical Studies for their part in handling and culturing the milk samples. 


\section{References}

Agumbah G.J.O., Ogaa J.S., Mutiga E.R., Muraguri J.M., 1983. Coliform mastitis in the Nairobi area of Kenya: bacteriological types, antimicrobial sensitivity patterns and clinical problems of treatment. Kenya Veterinarian, 7, 20-24.

Barkema, H. W., Shukkken, Y.H., Lam T. J. G. M., Beiboer, M. L., Benedictus, G., Brand, A. 1999. Management practices associated with clinical mastitis. . J.Dairy Sci, 82, 1643-1654.

Brown, M. B., Scasserra, A. E., 1990. Antimicrobial resistance in streptococcal species isolated from bovine mammary glands. Am. J.. Vet. Res., 51, 2015-2018.

Holt, J. G., Krieg, N.R., Sneath, P. H. A., Staley, J. T., and Williams, S. T., 1993. Bergeys Manual of Determinative Bacteriology, 9th ed, Williams and Wilkins, USA.

Gitau G. K., Waridi, M., Makame, H.A., Saleh, M.M., Muhamed, R.A., Mkola, A.P., Haji, M.A., 2003. Occurrence of high udder infection rates in dairy cows in Ungunja Island of Zanzibar, Tanzania. J. App. Res. Vet. Med., 1(1), 73-76.

De Oliveira, A. P., Watts J.L., Salmon, S.A., Aarestrup, F.M. 2000. Antimicrobial sensitivity of Staphylococcus aureus isolated from bovine mastitis in Europe and the United States. J. Dairy Sci.83, 855-862.

Erskine, R. J., Walker, R. D., Bolin, C. A., Bartlett, P. C., White, D. G. 2002. Trends in antimicrobial susceptibility of mastitis pathogens during a seven-year period. $J$. Dairy Sci, 85, 1111-1118.

Fang, W., and Pyorala, S. 1996. Mastitis-causing Escherichia coli: Serum sensitivity and susceptibility to selected antibacterials in milk. J. Dairy Sci, 79, 76-82.

Haile, T. 2004. Prevalence and factors associated with bovine mastitis in South Wollo, Ethiopia. Bull. Anim. Hith. Prod. Afr., 52, 1-6.

Miltenburg, J. D., de Lange, D., Crauwels, A.P.P., Bongers, J.H., Tielen, M.J.M., Schukken, Y.H., Elbers, A.R.W. 1996. Incidence of clinical mastitis in a random sample of dairy herds in the southern Netherlands, Vet. Rec., 139, 204-207.

Myllys, V., Asplund, K., Brofeldt, E., Hirvelae-Koski, V., Honkanen-Buzalski, T., Junttila, J., Kulkas, L., Myllykangas, O., Niskanen, M., Saloniemi, H., Sandholm, M.,

Radostitis, O. M. 1998 Herd health: Food Animal Production Medicine. 3rd Ed. W.B., Saunders Company.

Ethiop. Vet. J., 2011, 15 (1), 1-13 